\author{
Gianni Allegro* - Luca CRISTALDi* ${ }^{* *}$
}

\title{
Campionamenti di Carabidi nel Parco Fluviale del Po (tratto alessandrino, Piemonte) mediante trappole luminose a luce di Wood (Coleoptera Carabidae) $)^{1}$
}

\begin{abstract}
Riassunto: Un'indagine condotta negli anni 2014 e 2015 in quattro stazioni del Parco fluviale del Po (tratto alessandrino) utilizzando trappole luminose a luce di Wood ha consentito di censire 71 specie di Carabidi, 18 delle quali non segnalate nell'articolo di Allegro \& Sciaky (2001) relativo a questo stesso territorio. Vengono discusse le segnalazioni di alcune specie interessanti come Bembidion assimile, Lymnastis galilaeus, Egadroma marginatum, Acupalpus brunnipes, Trechicus nigriceps e Zuphium olens e, infine, i principali fattori limitanti relativi a questa tecnica di campionamento.

Abstract: Sampling of ground beetles (Coleoptera, Carabidae) by Wood light traps in the Po river natural park (eastern Piedmontese course). A survey carried out during 2014-2015 by Wood light traps in four different sites of the Piedmontese Po fluvial park (eastern course) showed the presence of 71 Carabid species, of which 18 were not previously recorded in the paper by Allegro \& Sciaky (2001) concerning the same fluvial area. The records of some interesting species such as Bembidion assimile, Lymnastis galilaeus, Egadroma marginatum, Acupalpus brunnipes, Trechicus nigriceps and Zuphium olens are discussed, as well as the main limiting factors of this sampling method.
\end{abstract}

Key words: Ground beetles, Light trap, Faunistics, Po river, Riparian habitats, Piedmont, Italy.

\section{INTRODUZIONE}

Gli ecosistemi fluviali sono particolarmente complessi e in genere molto ricchi di biodiversità, con flore e faune per lo più specifiche, talvolta minacciate per la progressiva rarefazione delle aree umide e paludose. In questi ambienti vengono frequentemente realizzati studi e monitoraggi indirizzati a migliorare le conoscenze sulle comunità animali e vegetali presenti al loro interno e a comprendere meglio i meccanismi del loro funzionamento (Moretti \& Patocchi, 2004), e in questo ambito vengono sovente studiate le comunità di Coleotteri Carabidi, che costituiscono ottimi bioindicatori perché in grado di fornire importanti indizi sulle condizioni ecologiche dei biotopi oggetto di studio (Thiele, 1977; Brandmayr et al., 2005).

La carabidofauna del Po piemontese era già stata oggetto di studi riguardanti sia il suo tratto cuneese (Della Beffa \& Rastelli, 2007) sia il tratto alessandrino (Allegro \& Sciaky, 2001), entrambi inseriti con Legge Regionale n. 28/1990 nel Sistema delle Aree protette della Fascia fluviale del Po. Della Beffa
\& Rastelli (2007), utilizzando tecniche tradizionali di ricerca (a vista o mediante trappole a caduta), hanno segnalato la presenza di 71 specie, mentre Allegro \& Sciaky (2001) ne hanno segnalate 187, distribuite nei principali ambienti ripari, diverse delle quali rare e molto interessanti.

Tuttavia, successive indagini condotte in altri ambienti piemontesi utilizzando una trappola luminosa a luce di Wood (Allegro, 2013) avevano messo in evidenza le grandi potenzialità di questa tecnica di campionamento, capace di portare alla luce specie di Carabidi mai precedentemente censite negli stessi territori. La trappola luminosa a luce di Wood è infatti molto efficace nella cattura di numerose specie di Coleotteri alate e dotate di elevata vagilità, ed è già stata utilizzata per migliorare le conoscenze faunistiche in diversi studi riguardanti i Coleotteri Carabidi (cfr. tra gli altri Kádár \& Lövei, 1992; Usis \& MacLean, 1998; Yahiro \& Yano, 1997).

Poiché le specie alate costituiscono una frazione elevata di quelle presenti negli ambienti igrofili e ripari

\footnotetext{
${ }^{*}$ Giani Allegro, CREA - Consiglio per la ricerca in agricoltura e l'analisi dell'economia agraria, PLF Unità di Ricerca per le Produzioni Legnose Fuori Foresta, Casale Monferrato (AL), Italia. E-mail: gianni.allegro@crea.gov.it

${ }^{* *}$ Luca Cristaldi, Parco del Po vercellese-alessandrino. E-mail: luca.cristaldi@parcodelpo-vcal.it

${ }^{1}$ Lavoro parzialmente realizzato con il contributo dell'Ente di gestione delle aree protette del Po vercellese-alessandrino nell'ambito del Progetto BassiBoschi.
} 
(lo stato alare macrottero è un carattere morfo-funzionale evolutivamente selezionato in ambienti instabili, come sono quelli ripari), questa metodologia è stata utilizzata in nuove indagini condotte in ambienti interni al Parco fluviale del Po piemontese nel suo tratto alessandrino, già interessato dallo studio di Allegro \& Sciaky (2001), allo scopo di migliorare le conoscenze sulla biodiversità entomatica di questo territorio.

\section{MATERIALI E METODI}

I campionamenti sono stati realizzati in quattro diverse località all'interno del Parco fluviale del Po, tratto alessandrino, tre delle quali su sponda de- stra del fiume Po in prossimità dei centri abitati di Valenza e di Bassignana (periodo 9/07-26/08/2014) e presso l'Azienda sperimentale Mezzi di Casale Monferrato, annessa all'Unità di Ricerca per le Produzioni legnose fuori foresta (CREA-PLF) (periodo 1/07-18/08/2015), e una su sponda sinistra del fiume all'interno della Garzaia di Valenza (periodo 1/0724/08/2015) (Fig. 1).

Le catture sono state realizzate con trappole luminose costituite da 2 tubi appaiati da $18 \mathrm{~W}$ a luce nera (luce di Wood) alimentati a rete, sotto le quali era collocato un secchio dotato superiormente di un imbuto che aveva lo scopo di impedire la fuga degli esemplari

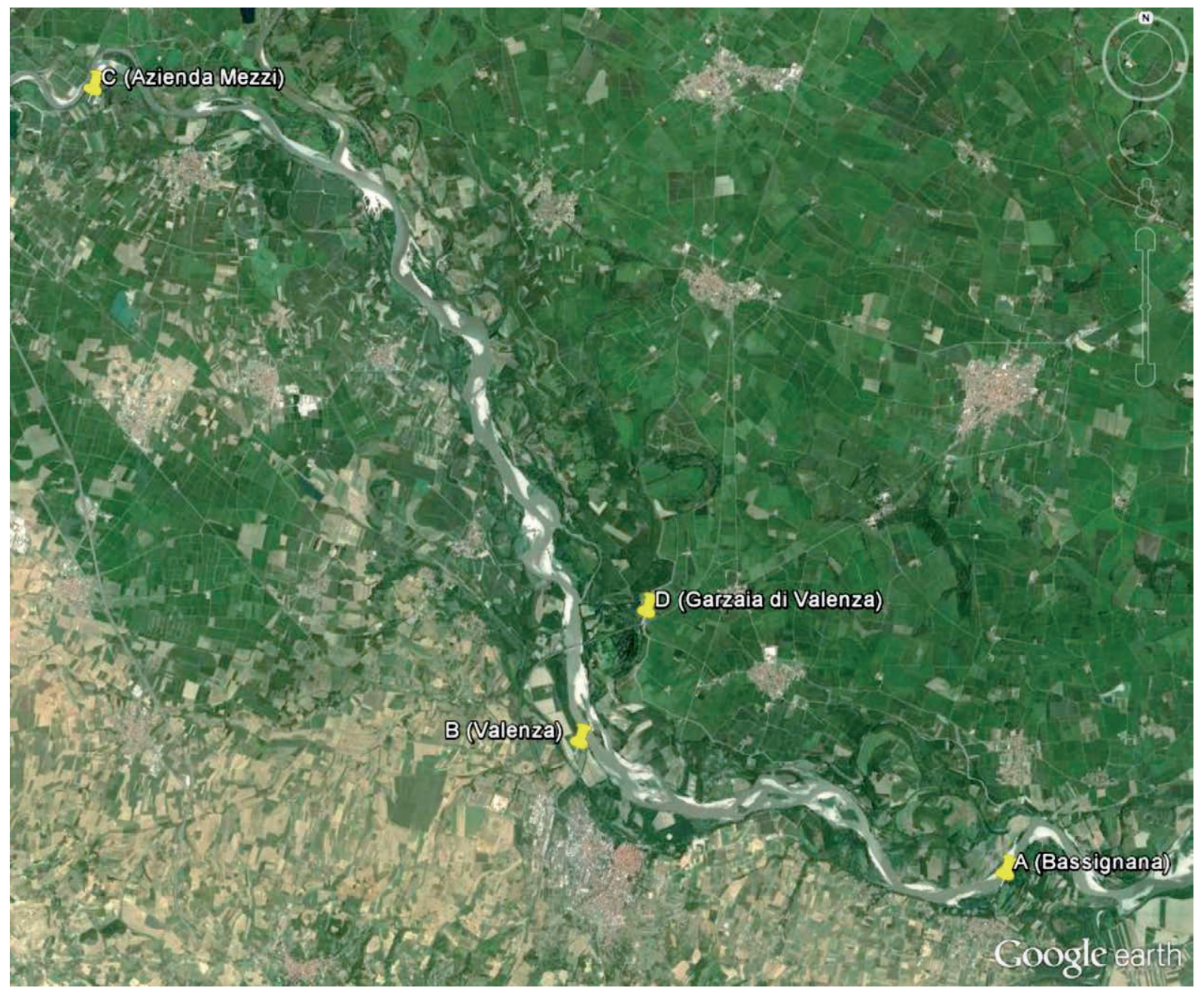

Fig. 1. Mappa da satellite delle quattro località di campionamento con trappola luminosa (immagine: Google Earth ${ }^{\circledR} 2013$ ). 
Tab. 1. Elenco delle specie di Carabidae catturate con trappola luminosa in quattro stazioni del Parco fluviale del Po tratto alessandrino ( $\mathrm{A}=\mathrm{Bassignana}, \mathrm{B}=$ Valenza, $\mathrm{C}=\mathrm{Az}$. Mezzi Casale Monf., $\mathrm{D}=\mathrm{Garzaia}$ di Valenza) con relative categoria corologica, preferenze ecologiche e frequenza di cattura (vedi testo). Sono contrassegnate con asterisco $\left(^{*}\right)$ quelle non segnalate nell'articolo di Allegro \& Sciaky (2001).

\begin{tabular}{|c|c|c|c|c|c|c|c|}
\hline Tribù & Specie & Corot. & Ecol. & A & B & $\mathrm{C}$ & $\mathrm{D}$ \\
\hline Brachinini & $\begin{array}{l}\text { Brachinus crepitans (Linnaeus, 1758) } \\
\text { Brachinus sclopeta (Fabricius, 1792) } \\
\text { Brachinus plagiatus Reiche, } 1868 * \\
\text { Brachinus psophia Audinet-Serville, } 1821\end{array}$ & $\begin{array}{l}\text { PAL } \\
\text { EUM } \\
\text { MED } \\
\text { TUE }\end{array}$ & $\begin{array}{l}\mathrm{X} \\
\mathrm{X} \\
\mathrm{I} \\
\mathrm{X}\end{array}$ & & $\begin{array}{l}+ \\
+ \\
+\end{array}$ & $\begin{array}{c}+ \\
++ \\
+\end{array}$ & \\
\hline Clivinini & $\begin{array}{l}\text { Clivina fossor (Linnaeus, 1758) } \\
\text { Clivina collaris (Herbst, 1784) }\end{array}$ & $\begin{array}{l}\text { OLA } \\
\text { TUE }\end{array}$ & $\begin{array}{l}\mathrm{X} \\
\mathrm{I}\end{array}$ & + & $\begin{array}{c}++ \\
+ \\
\end{array}$ & & \\
\hline Dyschiriini & $\begin{array}{l}\text { Dyschiriodes aeneus (Dejean, 1825) } \\
\text { Dyschiriodes nitidus (Dejean, 1825) } \\
\text { Dyschirius angustatus (Ahrens, 1830) } \\
\end{array}$ & $\begin{array}{l}\text { SIE } \\
\text { ASE } \\
\text { EUR }\end{array}$ & $\begin{array}{l}\text { I } \\
\text { I } \\
\text { I }\end{array}$ & + & $\begin{array}{c}++ \\
+ \\
\end{array}$ & & \\
\hline Bembidiini & $\begin{array}{l}\text { Bembidion laticolle (Duftschmid, 1812) } \\
\text { Bembidion striatum (Fabricius, 1792) } \\
\text { Bembidion dentellum (Thunberg, 1787) } \\
\text { Bembidion semipunctatum (Donovan, 1806) } \\
\text { Bembidion articulatum (Panzer, 1796) } \\
\text { Bembidion assimile Gyllenhal, 1810 } \\
\text { Bembidion latiplaga Chaudoir, 1850 } \\
\text { Bembidion lunulatum (Geoffroy in Fourcroy, 1795) } \\
\text { Bembidion quadrimaculatum (Linnaeus, 1761) } \\
\text { Bembidion quadripustulatum Audinet-Serville, 1821 } \\
\text { Bembidion testaceum (Duftschmid, 1812) } \\
\text { Paratachys bistriatus (Duftschmid, 1812) } \\
\text { Paratachys micros (Fischer von Waldheim, 1828) } \\
\text { Sphaerotachys hoemorrhoidalis (Ponza, 1805) } \\
\text { Lymnastis galilaeus Piochard de Brûlerie, 1876* }\end{array}$ & $\begin{array}{l}\text { EUR } \\
\text { SIE } \\
\text { EUR } \\
\text { PAL } \\
\text { ASE } \\
\text { WPA } \\
\text { MED } \\
\text { EUM } \\
\text { OLA } \\
\text { CEM } \\
\text { EUR } \\
\text { TUE } \\
\text { EUM } \\
\text { AFM } \\
\text { MED }\end{array}$ & $\begin{array}{l}\text { I } \\
\text { I } \\
\text { I } \\
\text { I } \\
\text { I } \\
\text { I } \\
\text { I } \\
\text { I } \\
\text { I } \\
\text { I } \\
\text { I } \\
\text { I } \\
\text { I } \\
\text { I } \\
\text { I }\end{array}$ & $\begin{array}{l}++ \\
++ \\
++ \\
+\end{array}$ & $\begin{array}{c}++ \\
++ \\
++ \\
++ \\
+ \\
++ \\
++ \\
+ \\
+\end{array}$ & $\begin{array}{c}+ \\
++ \\
+ \\
+++ \\
+\end{array}$ & + \\
\hline Trechini & $\begin{array}{l}\text { Blemus discus discus (Fabricius, 1792) } \\
\text { Perileptus areolatus (Creutzer, 1799) }\end{array}$ & $\begin{array}{l}\text { OLA } \\
\text { EUM }\end{array}$ & $\begin{array}{l}\text { I } \\
\text { I }\end{array}$ & ++ & $\begin{array}{c}++ \\
+ \\
\end{array}$ & + & ++ \\
\hline Pterostichini & $\begin{array}{l}\text { Pterostichus aterrimus aterrimus (Herbst, 1784) } \\
\text { Pterostichus niger niger (Schaller, 1783) } \\
\text { Pterostichus oenotrius } \text { Ravizza, 1975 }\end{array}$ & $\begin{array}{l}\text { WPA } \\
\text { ASE } \\
\text { SEU }\end{array}$ & $\begin{array}{l}\text { I } \\
\text { I } \\
\text { I }\end{array}$ & & + & $\begin{array}{l}+ \\
+\end{array}$ & + \\
\hline Zabrini & Amara bifrons (Gyllenhal, 1810) & CAE & $\mathrm{X}$ & & & + & \\
\hline Anisodactylini & $\begin{array}{l}\text { Anisodactylus signatus (Panzer, 1796) } \\
\text { Scybalicus oblongiusculus (Dejean, 1829) }\end{array}$ & $\begin{array}{l}\text { ASE } \\
\text { MED } \\
\end{array}$ & $\begin{array}{l}\mathrm{I} \\
\mathrm{X} \\
\end{array}$ & + & $\begin{array}{c}++ \\
++ \\
+\end{array}$ & $\begin{array}{c}++ \\
+ \\
\end{array}$ & ++ \\
\hline Harpalini & $\begin{array}{l}\text { Pseudoophonus rufipes (Degeer, 1774) } \\
\text { Pseudoophonus griseus (Panzer, 1796) } \\
\text { Pseudoophonus calceatus (Duftschmid, 1812)* } \\
\text { Parophonus hirsutulus (Dejean, 1829) } \\
\text { Parophonus maculicornis (Duftschmid, 1812) } \\
\text { Harpalus anxius (Duftschmid, 1812) } \\
\text { Harpalus froelichii Sturm, 1818 } \\
\text { Harpalus smaragdinus (Duftschmid, 1812) } \\
\text { Cryptophonus tenebrosus (Dejean, 1829)* } \\
\text { Ophonus ardosiacus (Lutshnik, 1922) * } \\
\text { Ophonus diffinis (Dejean, 1829)* } \\
\text { Ophonus rufibarbis (Fabricius, 1792) } \\
\text { Ophonus sabulicola (Panzer, 1796) * } \\
\text { Ophonus azureus (Fabricius, 1775) } \\
\text { Ophonus puncticeps Stephens, 1828 * } \\
\text { Ophonus melletii (Heer, 1837)* } \\
\text { Amblystomus niger (Heer, 1841) }\end{array}$ & $\begin{array}{l}\text { OLA } \\
\text { PAL } \\
\text { ASE } \\
\text { TUM } \\
\text { SEU } \\
\text { PAL } \\
\text { ASE } \\
\text { TEM } \\
\text { WPA } \\
\text { EUM } \\
\text { EUR } \\
\text { TEM } \\
\text { TUE } \\
\text { CEM } \\
\text { TUE } \\
\text { EUR } \\
\text { EUM }\end{array}$ & $\begin{array}{l}X \\
X \\
X \\
I \\
X \\
X \\
X \\
X \\
X \\
X \\
X \\
X \\
X \\
X \\
X \\
X \\
X\end{array}$ & $\begin{array}{c}+++ \\
+++ \\
++ \\
+++ \\
+ \\
+ \\
+++ \\
++ \\
+ \\
+ \\
+++\end{array}$ & $\begin{array}{c}+++ \\
+++ \\
++ \\
++ \\
++ \\
+ \\
+ \\
++ \\
++\end{array}$ & $\begin{array}{c}+++ \\
++ \\
+++ \\
+ \\
+++ \\
+ \\
+ \\
+\end{array}$ & $\begin{array}{c}+ \\
+++ \\
+\end{array}$ \\
\hline
\end{tabular}


Tab. 1. Segue dalla pagina precedente

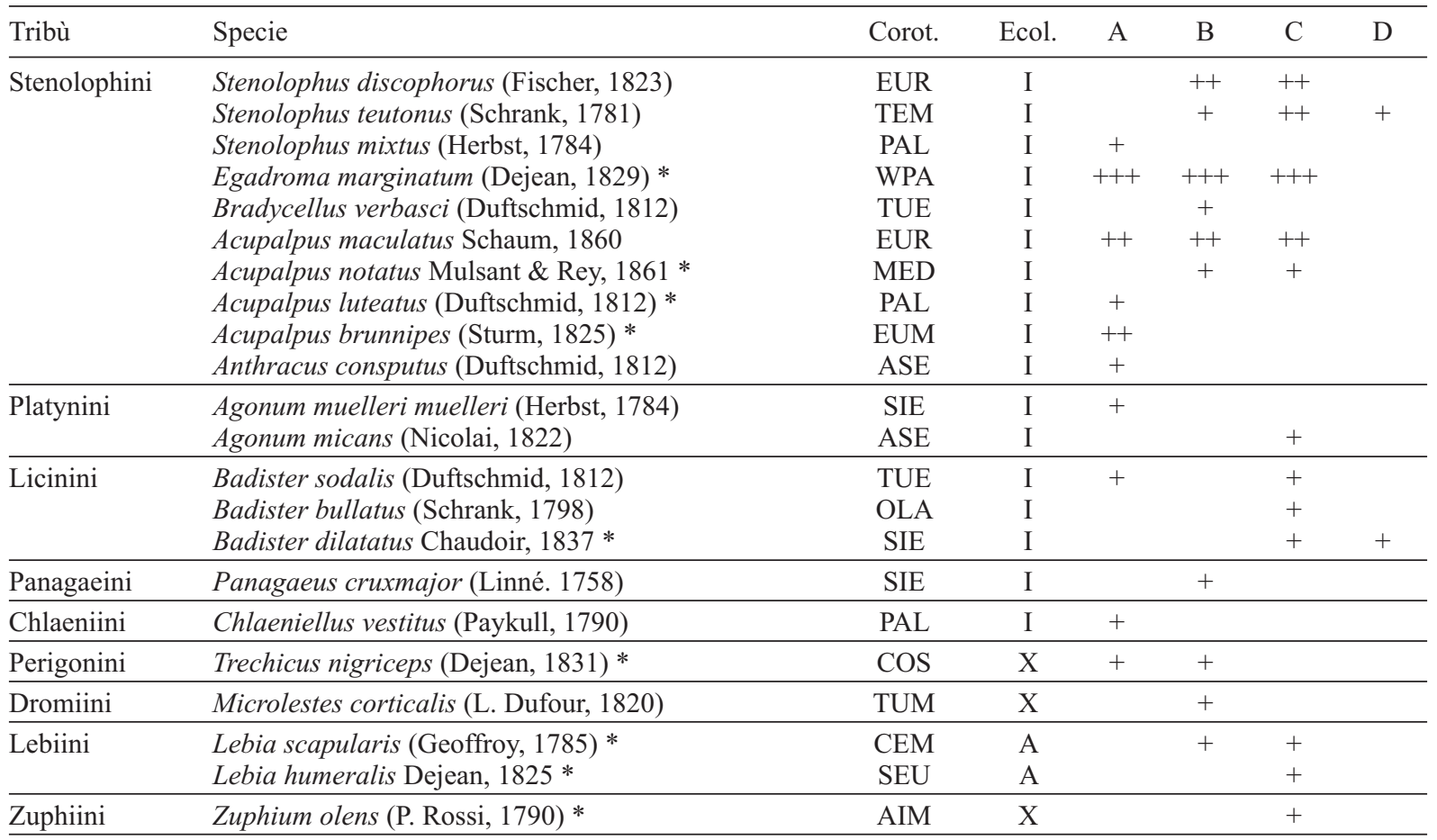

caduti al suo interno. La conservazione degli esemplari era assicurata da una soluzione sovrasatura di acqua e sale da cucina contenuta all'interno del secchio.

Tutti gli esemplari di Carabidi catturati sono stati identificati a livello di specie. Alcuni esemplari sono stati preparati a secco su cartellino, mentre i rimanenti sono conservati in alcool etilico $70 \%$ all'interno di provette nel laboratorio dei Entomologia del CREA-PLF.

Di tutte le specie censite vengono fornite informazioni sulla rispettiva categoria corologica sensu Vigna Taglianti (2005a), le preferenze ecologiche (I=igrofila; $\mathrm{X}=$ xerotermofila; $\mathrm{A}=$ arboricola) e l'abbondanza delle catture realizzate nelle quattro località nel corso della presente indagine (+ sporadiche; ++ comuni; +++ abbondanti). La nomenclatura delle specie è quella riportata nella Checklist di Vigna Taglianti (2005b) tranne che per i Bembidiina, per i quali si è assunta come base la più recente checklist di Neri et al. (2011).

\section{RISULTATI}

In Tab. 1 sono elencate le 71 specie di Carabidi censite, 18 delle quali, contrassegnate da asterisco $(*)$, non erano segnalate nell' articolo di Allegro \& Sciaky (2001) riguardante lo stesso territorio. Esse vengono presentate per tribù di appartenenza, con le già citate informazioni su corotipo, preferenze ecologiche e abbondanza di cattura.

Tra le nuove specie censite alcune sono molto rare in territorio piemontese: alcune di queste ( $\mathrm{Lym}$ nastis galilaeus, Egadroma marginatum) sono state segnalate per la prima volta in Piemonte solo molto recentemente (Allegro, 2013), mentre un'altra ( $\mathrm{Acu}$ palpus brunnipes) era nota per una sola segnalazione recente in regione (Pescarolo, 1994). Si tratta di specie fortemente igrofile e paludicole, di piccole o piccolissime dimensioni, difficilmente censibili con metodi di ricerca a vista. Altre specie tra quelle non censite da Allegro \& Sciaky (2001) sono connotate da elevata igrofilia, come ad esempio Brachinus plagiatus, $\mathrm{Ba}$ dister dilatatus, Acupalpus notatus e A. luteatus. Diverse altre sono invece connotate da abitudini tendenzialmente termofile e steppiche (Pseudoophonus calceatus, Cryptophonus tenebrosus, i diversi 
Ophonus, Trechicus nigriceps e Zuphium olens). Due specie soltanto hanno costumi arboricoli (Lebia scapularis e L. humeralis).

Vengono di seguito riportate alcune informazioni di carattere ecologico e distributivo relative alle specie di maggiore interesse.

\section{Bembidion assimile Gyllenhal, 1810 (Fig. 2)}

Specie luticola già segnalata da Allegro \& Sciaky (2001) di Alluvioni Cambiò, ma molto rara in tutta la Pianura Padana. Durante l'indagine ne è stato catturato un singolo esemplare nella Garzaia di Valenza.

Lymnastis galilaeus Piochard de la Brûlerie, 1876

Specie solo recentemente segnalata in Piemonte (Allegro, 2013). Ripicola e limicola, è caratterizzata da spiccato fototropismo positivo, specialmente per quel che riguarda il sesso femminile (Magrini, 2010); le rare catture degli individui di que-

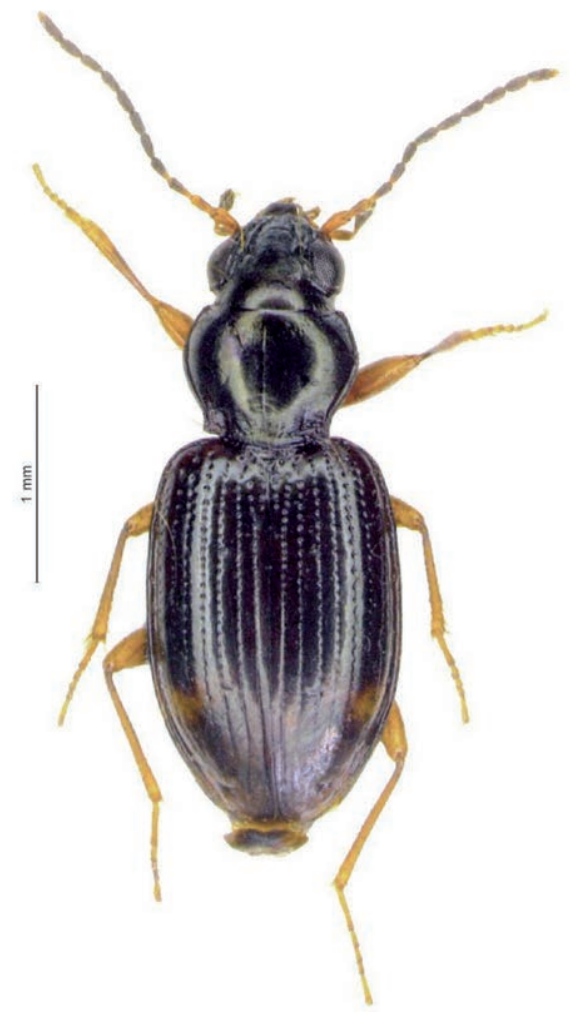

Fig. 2. Bembidion assimile Gyllenhal, 1810 (foto Gianni Allegro). sta specie riportate in letteratura sono infatti state per lo più realizzate con trappole luminose.

Egadroma marginatum (Dejean, 1829) (Fig. 3)

Specie ripicola e paludicola da poco segnalata in Piemonte (Allegro, 2013), oggi frequentemente catturata, anche in abbondanza, negli studi condotti dal primo autore con trappola luminosa a luce di Wood (cfr. Allegro, 2015). Nella presente indagine essa è stata catturata abbondante in tutte le stazioni sottoposte a campionamento.

\section{Acupalpus brunnipes (Sturm, 1825) (Fig. 4)}

Indicato da Baudi di Selve (1889) e Della Beffa (1912) come raro in territorio piemontese e da Luigioni (1929) come presente in quasi tutte le regioni d'Italia, compreso il Piemonte. L'unica recente segnalazione in regione riguarda la Baraggia di Piano Rosa (Pescarolo, 1994). È anch'essa specie spiccatamente igrofila.

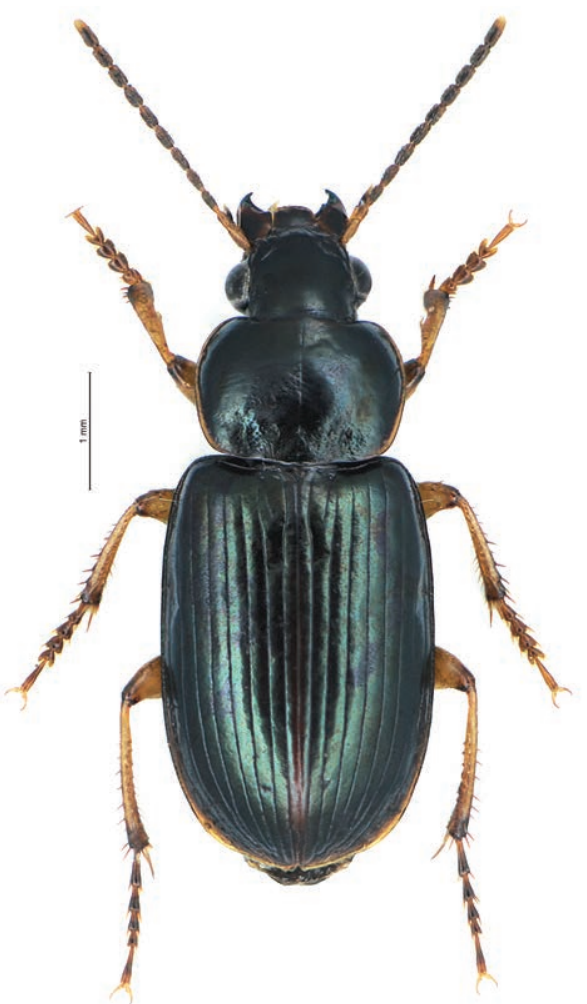

Fig. 3. Egadroma marginatum (Dejean, 1829) (foto Andrea Carlin). 
Trechicus nigriceps (Dejean, 1831)

Specie non rara ma molto localizzata in Piemonte. Originariamente a distribuzione intertropicale, oggi può essere considerata subcosmopolita per trasporto antropico attraverso le vie di scambio commerciale. Già citata da Magistretti (1965) di alcune altre località piemontesi e recentemente segnalata da Allegro $(2013,2015)$ per il Monferrato.

\section{Zuphium olens (P. Rossi, 1790)}

Specie dalla biologia ancora poco conosciuta, forse meno rara di quanto supposto in passato perché frequentemente censita negli studi condotti con trappola luminosa (cfr. Allegro, 2010 e 2015).

\section{CONCLUSIONI}

L'impiego della lampada a luce di Wood ha consentito un significativo aumento delle conoscenze faunistiche relative alle specie di Coleotteri Carabidi presenti nel territorio del Parco fluviale del Po (tratto alessandrino). Alle 187 specie di Carabidi già censite

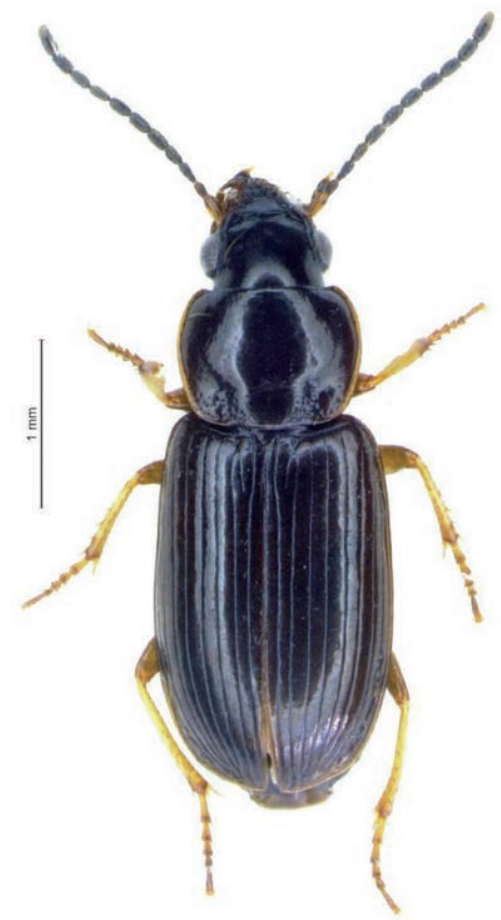

Fig. 4. Acupalpus brunnipes (Sturm, 1825) (foto Gianni Allegro). da Allegro \& Sciaky (2001) se ne aggiungono infatti ulteriori 18 , alcune delle quali rare in tutto il territorio padano, che erano sfuggite ai metodi di indagine tradizionali. L'elevato numero delle specie censite nel presente studio (71), unicamente condotto con trappola luminosa, sottolinea le grandi potenzialità di questo strumento, che soffre tuttavia di alcune limitazioni.

In primo luogo la lampada, soprattutto se collocata in posizione elevata e ben visibile dalla distanza, possiede un ampio raggio d'azione ed è difficile pertanto l'attribuzione delle specie catturate a uno specifico biotopo, soprattutto se questo presenta dimensioni limitate.

Inoltre l'efficacia della trappola a luce di Wood risente in modo determinante delle condizioni atmosferiche, risultando massima nelle notti calde e afose in assenza di vento e al contrario quasi nulla, almeno per quanto riguarda i Carabidi, con temperature notturne inferiori a $20^{\circ} \mathrm{C}$ o in presenza di ventilazione. Per questo motivo le catture più interessanti sono in genere possibili soltanto nei mesi estivi: il mese di luglio, anche in relazione ai cicli biologici delle specie, sembra presentare le condizioni più favorevoli per queste indagini in ambiente padano, mentre durante il mese di agosto, pur perdurando condizioni climatiche favorevoli, si assiste in genere a un impoverimento e a una banalizzazione della fauna censita.

Un ulteriore fattore limitante è costituito dalla variabilità della sensibilità specifica verso la sorgente luminosa: accanto a specie che vengono costantemente attratte in gran numero (per esempio negli ambienti cui fa riferimento il presente studio sono molto abbondanti le catture di Harpalus froelichii, Egadroma marginatum, Parophonus hirsutulus e Pseudoophonus spp.) ve ne sono altre, anch'esse alate e comuni negli ambienti ripari, che vengono raramente catturate con la trappola luminosa. Per esempio, tra le numerose specie appartenenti al genere Amara sicuramente presenti lungo le rive del Po, soltanto Amara bifrons (Gyllenhal, 1810) è stata censita nel corso della presente indagine. E ancora, le specie del genere Agonum appartenenti al sottogenere Melanagonum non vengono attratte, mentre vengono sporadicamente attratti Agonum (s. str.) muelleri (Herbst, 1784) e le specie del sottogenere Europhilus.

Ma la limitazione più importante è ovviamente il fatto che la trappola luminosa non ha alcuna possibilità di censire le specie microttere incapaci di spo- 
starsi in volo, che sono sovente quelle caratterizzate dai livelli più elevati di specializzazione verso gli ambienti ecologicamente più stabili, come ad esempio le foreste, e sono pertanto quelle dotate del maggior potere di bioindicazione (quanto meno relativamente a tali ambienti). A questo proposito è interessante il tentativo da parte di alcuni ricercatori (Hébert et al., 2000) di abbinare una sorgente luminosa attrattiva alla tradizionale trappola a caduta (pitfall trap), con risultati interessanti per la maggior accuratezza e completezza ottenibili nei censimenti faunistici, ma anche con un sensibile aggravio dei costi e della complessità delle operazioni di campionamento.

Ciò non toglie che la trappola luminosa a luce di Wood possa costituire un utile supporto negli studi faunistici condotti su vasta scala territoriale, inte- grando i metodi di ricerca tradizionali, per migliorare le conoscenze e per censire in particolare le specie igrofile e riparie oppure quelle termofile e steppiche, per la maggior parte alate e dotate di elevata vagilità, alcune delle quali difficilmente osservabili con i metodi tradizionali perché caratterizzate da biologia o da preferenze ecologiche particolari.

\section{RINGRAZIAMENTI}

Si ringraziano il Responsabile della Vigilanza del Parco Carlo Carbonero per il supporto alla sistemazione delle trappole, Federico Felli dell'Agriturismo Cascina Nuova di Valenza per il supporto logistico, Achille Casale e Riccardo Sciaky per la revisione del manoscritto e Andrea Carlin per la concessione dell'immagine di Egadroma marginatum.

\section{BIBLIOGRAFIA}

Allegro G., Sciaky R., 2001 - I Coleotteri Carabidi del Po piemontese (tratto orientale). Bollettino del Museo regionale di Scienze naturali di Torino, 18(1): 173-201.

Allegro G., 2010 - I Carabidi del Basso Monferrato (Italia - Piemonte) (Coleoptera: Carabidae). In Picco F. (a cura di) 'Nascitur in Collibus Montisferrati. Biodiversità del Basso Monferrato'. Diffusioni Grafiche, Villanova Monf.: 103-114.

Allegro G., 2013 - Nuove segnalazioni di Carabidi per il Piemonte e considerazioni sulla carabidofauna piemontese (Coleoptera Carabidae). Rivista Piemontese di Storia Naturale, 34: 171-180.

Allegro G., 2015 - La fauna carabidica dell'Alneto del Mulino (Piemonte, Nord-Ovest Astigiano) (Coleoptera, Carabidae). I Quaderni di Muscandia, 14(2014): 109-117.

Baudi di Selve F., 1889- Catalogo dei Coleotteri del Piemonte. Annali della Regia Accademia di Agricoltura di Torino, 32 : $51-274$.

Brandmayr P., Zetto T., Pizzolotto R. (a cura di), 2005 - I Coleotteri Carabidi per la valutazione ambientale e la conservazione della biodiversità. Manuale operativo. APAT, Manuali e linee guida, 34: $240 \mathrm{pp}$.

Della Beffa G., 1912 - I Coleotteri dell'agro torinese e loro rapporti colla vegetazione e l'agricoltura. Annali della regia Accademia di Agricoltura di Torino, 54 (1911): 69-346.

Della Beffa G., Rastelli M., 2007 - Le cenosi carabidologiche delle sponde del fiume Po nel Parco del Po Cuneese (Coleoptera, Carabidae). In: Delmastro G.B., Gaggino A., Giachino P.M., Morisi A., Rastelli M. (eds.). Ricerche sugli ambienti acquatici del Po Cuneese - Interreg IIIA “Aqua”. Memorie dell’Associazione Naturalistica Piemontese, 8: 97-110.

Hébert C., Jobin L., Fréchette M., Pelletier G., Coulombe C., Germain C., Auger M., 2000 - An efficient pit-light trap to study beetle diversity. Journal of Insect Conservation, 4: 191-202.

KÁdÁR F., Lövei G.L., 1992 - Light trapping of Carabids (Coleoptera: Carabidae) in an apple orchard in Hungary. Acta Phytopathologica et Entomologica Hungarica, 27(1-4): 343-348.

Luigioni P., 1929 - I Coleotteri d'Italia. Catalogo sinonimico-topografico-bibliografico. Memorie della pontificia Accademia delle Scienze "I nuovi Lincei”, 2(13): 1160 pp.

MAGRINi P., 2010 - Revisione delle specie europee appartenenti al Genere Lymnastis Motschulsky, 1862 con descrizione di tre nuovi taxa (Coleoptera, Carabidae, Trechinae). Annali del Museo Civico di Storia Naturale "G. Doria”, 102: 337-390.

Moretti M., Patocchi N., 2004 - Bioindicazione faunistica in ecosistemi fluviali ripari. Principi e metodologie proposte nell'ambito di un programma di controllo a lungo termine delle golene svizzere. Studi Trentini di Scienze Naturali, Acta Biologica, 80 (2003): 209-215.

Neri P., Bonavita P., Gudenzi I., Magrini P., Toledano L., 2011 - Bembidiina della fauna italo-corsa: chiavi di identificazione (Insecta Coleoptera Carabidae). Quaderno di Studi e Notizie di Storia Naturale della Romagna, 33: 1-183.

Pescarolo R., 1994 - I Coleotteri Carabidi della Baraggia di Piano Rosa (Piemonte, Novara). Rivista Piemontese di Storia Naturale, 14(1993): 171-183. 
Thiele H.U., 1977 - Carabid beetles in their environments. Springer Verlag, Berlin, 369 pp.

Usis J.D., MacLean D.B., 1998 - The Ground Beetles (Coleoptera: Carabidae) of Stillfork Swamp Nature Preserve, Carrol County, Ohio. The Ohio Journal of Science, 98(4-5): 66-68.

Vigna TAglianti A., 2005a - Distribuzione geografica e tipi corologici dei Carabidi italiani. 3.5. In: BRANDMAYR P., ZETTO T., Pizzolotto R. (eds), I Coleotteri Carabidi per la valutazione ambientale e la conservazione della biodiversità. Manuale operativo. APAT, Manuali e linee guida, 34: p. 74-82.

Vigna TAglianti A., 2005b - Checklist e corotipi delle specie di Carabidae della fauna italiana. Appendice B. In: BRANDMAYR P., Zetto T., Pizzolotto R. (eds), I Coleotteri Carabidi per la valutazione ambientale e la conservazione della biodiversità. Manuale operativo. APAT, Manuali e linee guida, 34: p. 186-225.

YAHIRO K., YANO K., 1997 - Ground beetles (Coleoptera, Caraboidea) caught by a light trap during ten years. Esakia, $37: 57-69$. 